\title{
Study of the Role of Salivary Lactate Dehydrogenase in Habitual Tobacco Chewers, Oral Submucous Fibrosis and Oral Cancer as a Biomarker
}

\author{
Tejashree Mantri ${ }^{1}$, Sanjay G Thete ${ }^{2}$, Vijayalaxmi Male ${ }^{3}$, Renu Yadav ${ }^{4}$, Ishita Grover ${ }^{5}$, Girija R Adsure ${ }^{6}$, Dinraj Kulkarni ${ }^{7}$
}

\begin{abstract}
Aim: To evaluate the salivary lactate dehydrogenase (LDH) levels in clinico-pathologically confirmed oral submucous fibrosis (OSMF), oral cancer and clinically diagnosed tobacco pouch keratosis patients.

Materials and methods: A prospective, comparative study was carried out in a tertiary healthcare centre located in Loni from October 2013 to January 2014. A total of 120 patients were separated into 4 groups depending upon the clinical diagnosis as follows. Group I: healthy control (with no addictions and diseases). Group II: oral cancer. Group III: oral submucous fibrosis. Group IV: habitual tobacco chewers (tobacco addiction without any disease). Substantiation was done using biopsy. The samples were inspected for salivary LDH levels by the technique in line with the recommendations of the International Federation of Clinical Chemistry with the help of Erba Chem semi auto analyser.

Results: The mean salivary LDH levels in the control, oral cancer OSMF and habitual tobacco chewer group were $86.12 \pm 7.05 \mathrm{IU} / \mathrm{L}, 592.09 \pm 28.57$ $\mathrm{IU} / \mathrm{L}, 350.43 \pm 5.90 \mathrm{IU} / \mathrm{L}$ and $125.19 \pm 13.42 \mathrm{IU} / \mathrm{L}$, respectively. Out of 4 groups, LDH activity was increased in saliva of patients with tobacco pouch keratosis, OSMF, and oral cancer consistently. Notable difference was found in the mean salivary levels of the above groups. Results were subjected to appropriate statistical analysis: one-way ANOVA, Student's unpaired $t$ test for group-wise comparison followed by post hoc Tukey's test.
\end{abstract}

Conclusion: We observed congruous higher levels of salivary LDH in oral precancer and cancer, and hence it could be a future marker. Keywords: Lactate dehydrogenase, Oral cancer, Oral submucous fibrosis, Saliva, Tobacco pouch keratosis.

The Journal of Contemporary Dental Practice (2019): 10.5005/jp-journals-10024-2619

\section{INTRODUCTION}

The enzyme LDH is found in all living cells. Increased LDH activity is considered as a marker of cellular necrosis, but oral epitheliumshedding cells could be the major source of salivary LDH. ${ }^{1}$

Many methods are available today for diagnosis of cancers, but more emphasis is always given to a noninvasive and accurate test for diagnosis; thus saliva, an important and potential biomarker, can be used as an adjunctive step for diagnosing oral cancers and precancers, which can improve the prognosis and outcome of the disease process. ${ }^{2}$

Habitually chewing tobacco leaves results in the development of a well-recognized white mucosal lesion in the area of tobacco contact, called tobacco pouch keratosis; they are significantly different from true leukoplakia and have a much lower risk of malignant transformation. ${ }^{3}$

A precancerous condition (or premalignant condition) is a generalized state in which there is an increased risk of cancer when compared to the normal counterpart. If left untreated, these conditions may lead to cancer. Some of the premalignant conditions are leukoplakia, erythroplakia, lichen planus and OSMF.

Oral submucous fibrosis is a chronic, debilitating disease characterized by inflammation and progressive fibrosis of the submucosal tissues (lamina propria and deeper connective tissues). The condition is well recognized for its malignant potential and is particularly associated with areca nut chewing, the main component of betel quid. The pathogenesis of OSMF is not well established and believed to be multifactorial. The cascade begins with a juxtaepithelial inflammatory reaction in the oral mucosa, which ultimately results in trismus. ${ }^{4}$

\begin{abstract}
${ }^{1,3}$ Department of Oral Medicine and Radiology, Maharashtra Institute of Dental Sciences and Research, Latur, Maharashtra, India

${ }^{2,4}$ Department of Oral Pathology and Microbiology, Rural Dental College, Pravara Institute of Medical Sciences (Deemed University) Loni, Maharashtra, India

${ }^{5}$ Department of Prosthodontics and Implantology, M.A. Rangoonwala College of Dental Sciences and Research Center, Pune, Maharashtra, India ${ }^{6}$ Department of Dentofacial Orthopedics and Orthodontics, M.A. Rangoonwala College of Dental Sciences and Research Institute, Pune, Maharashtra, India

${ }^{7}$ Department of Oral and Maxillofacial Pathology, M.A. Rangoonwala College of Dental Sciences and Research Institute, Pune, Maharashtra,
\end{abstract} India

Corresponding Author: Tejashree Mantri, Department of Oral Medicine and Radiology, Maharashtra Institute of Dental Sciences and Research, Latur, Maharashtra, India, Phone: +91 8169932296, e-mail: mantritejashree@gmail.com

How to cite this article: Mantri T, Thete SG, et al. Study of the Role of Salivary Lactate Dehydrogenase in Habitual Tobacco Chewers, Oral Submucous Fibrosis and Oral Cancer as a Biomarker. J Contemp Dent Pract 2019;20(8):970-973.

Source of support: Nil

Conflict of interest: None

Oral squamous cell carcinoma is considered as the 18th most common cancer worldwide and 3rd most common cancer in India. Despite significant improvements in cancer diagnoses and treatments developed over the past few decades, early detection

(c) The Author(s). 2019 Open Access This article is distributed under the terms of the Creative Commons Attribution 4.0 International License (https://creativecommons. org/licenses/by-nc/4.0/), which permits unrestricted use, distribution, and non-commercial reproduction in any medium, provided you give appropriate credit to the original author(s) and the source, provide a link to the Creative Commons license, and indicate if changes were made. The Creative Commons Public Domain Dedication waiver (http://creativecommons.org/publicdomain/zero/1.0/) applies to the data made available in this article, unless otherwise stated. 
of cancer through diagnostic, prognostic and predictive biomarkers represents one of the most promising research fields for identifying early-stage cancer and personalizing therapies. Till date there are only a handful of studies being performed successively in OSCC patients by using saliva as the tumor biomarker. The saliva comes in direct contact with oral cancer lesions and hence it gives great benefits and accurate diagnosis. ${ }^{5}$

Tumor markers have gained much attention recently in early diagnosis and treatment of head and neck cancer. Blood is considered as one of the media of choice of all body fluids, to study biochemical markers in the medical field. Though it is considered as a medium of choice, it has its own constitutive disadvantages. ${ }^{6}$

Despite the disadvantages, a growing number of researchers are finding that saliva provides an easily available, noninvasive diagnostic medium for a rapidly widening range of diseases and clinical situations. ${ }^{7}$

Therefore, the present study was put through to determine the changes in the salivary alterations in LDH levels in OSMF, habitual tobacco chewers, oral cancer $(\mathrm{OC})$ and healthy controls and to explore the possibility of using LDH as a biochemical marker in the above-mentioned conditions as a reliable diagnostic aid.

\section{Materials and Methods}

This prospective study was conducted in patients clinically diagnosed with tobacco pouch keratosis and clinico-pathologically confirmed oral squamous cell carcinoma and OSMF in the age group of 18-70 years irrespective of gender reporting to the Department of Oral Medicine and Radiology at Rural Dental College and Hospital, Loni.

The subjects were briefed about the study and informed consent was obtained. Ethical committee approval was obtained.

Patients with a history of previous malignancy, history of treated oral cancer and OSMF and with a history of myocardial infarction, liver disease, renal disease and muscle dystrophy known to increase serum LDH levels were excluded from the study.

Selected patients were grouped as follows: group I: healthy controls (with no addictions and diseases), group II: habitual tobacco chewers (tobacco addiction without any disease), group III: oral submucous fibrosis, and group IV: oral cancer.

Unstimulated whole saliva measuring approximately one millilitre was collected from each of these patients by spit method in the calibrated measuring cup, usually once every 60 seconds for 5-15 minutes then transferred to a test tube and was centrifuged for 5 minutes at $5000 \mathrm{rpm}$.

Due care was taken to ensure that patients do not consume food, smoke or chew gum for at least one hour before the saliva collection procedure.

An LDH-P kit (Erba Mannheim obtained from Transasia BioMedicals Ltd, Solen India) was used to carry out the assay. LDH were evaluated by an UV semiautomated spectrophotometer. The assay was carried out within 24 hours as LDH in saliva is stable for 24 hours. Samples were kept cold after collection $\left(4^{\circ} \mathrm{C}\right)$ before evaluation. As per procedural guidelines provided in the leaflet of the kit, the working reagent and the sample were mixed well and aspirated, and then the readings were recorded.

\section{Statistics}

One-way ANOVA and chi square tests were used for multiple group comparison followed by post hoc Tukey's test for groupwise comparison.
Systat software version 12 (by Cranes Software, Bengaluru) was used.

\section{Results}

A prospective, comparative clinical study was conducted comprising 4 groups including 120 patients who are classified depending upon the clinical diagnosis as follows:

Group I: healthy control-it consisted of age- and sex-matched healthy subjects, who are not having oral cancer, precancer and history of any major illness in the past and present.

Group II: oral cancer-it comprises 30 subjects with welldifferentiated to poorly differentiated oral squamous cell carcinoma having clinicopathological correlation.

Group III: oral submucous fibrosis-it includes 30 subjects with grade I-grade IV OSMF having clinicopathological correlation.

Group IV: habitual tobacco chewers (tobacco addiction without any disease) - it contains 30 subjects with clinical correlation.

Levels of the enzyme activity in 4 groups were compared.

Comparison of the mean and standard deviation (SD) values of LDH IU/L was done between healthy control group, oral cancer group, oral submucous fibrosis group, and habitual tobacco chewers group, and the values were $86.12 \pm 7.05 \mathrm{IU} / \mathrm{L}, 592.09 \pm$ $28.57 \mathrm{IU} / \mathrm{L}, 350.43 \pm 5.90 \mathrm{IU} / \mathrm{L}$, and $125.19 \pm 13.42 \mathrm{IU} / \mathrm{L}$, respectively (Fig. 1, Table 1). The highest mean LDH value amongst all the cases obtained was in oral cancer patients.

By applying the one-way ANOVA test for repeated measures, the variation among column means are found to be significantly greater than expected and by applying Tukey-Kramer multiple comparison test there is a highly significant difference between the mean values of LDH IU/L in healthy control, oral cancer, OSMF, and habitual tobacco chewers groups compared to each other $(p<0.001)$.

The value of $F$ was $6056.2, p<0.001$, which is highly significant (Table 2).

\section{Discussion}

The major risk factor for tobacco pouch keratosis, OSMF, and oral cancer is the chewing of betel quid containing areca nut. The amount of areca nut in betel quid and the frequency and duration of chewing betel quid are clearly related to the development of the above-mentioned lesions. Other factors such as genetic and immunologic predisposition probably also play role. Betel nut extracts increase the rate of cell division, reduce the cell cycle time, induce DNA strand breaks, and induce unscheduled DNA synthesis. ${ }^{8}$

It is a multistage process from normal to dysplastic lesions and ultimately to squamous cell carcinoma. ${ }^{9}$ These dysplastic changes are associated with high glycolytic activity with a shift from aerobic to anaerobic glycolysis, resulting in a concomitant increase in lactate dehydrogenase (LDH) enzyme activity which can be demonstrated in tissue fluids and sera ${ }^{10}$. High glycolytic activity leads to production of a large quantity of lactic acid. Lactic acid dehydrogenase (LDH) is considered a key enzyme in glycolysis. It catalyses the process of lactate production in pirogronian reduction reaction.

Gradual conversion of normal tissue to premalignant lesion and subsequently to oral cancer which results in the alteration of the glycolytic pathway and hence the LDH levels. ${ }^{11}$

The present study was executed to evaluate the salivary LDH levels in patients with oral cancer and OSMF, habitual tobacco chewers and healthy controls. 


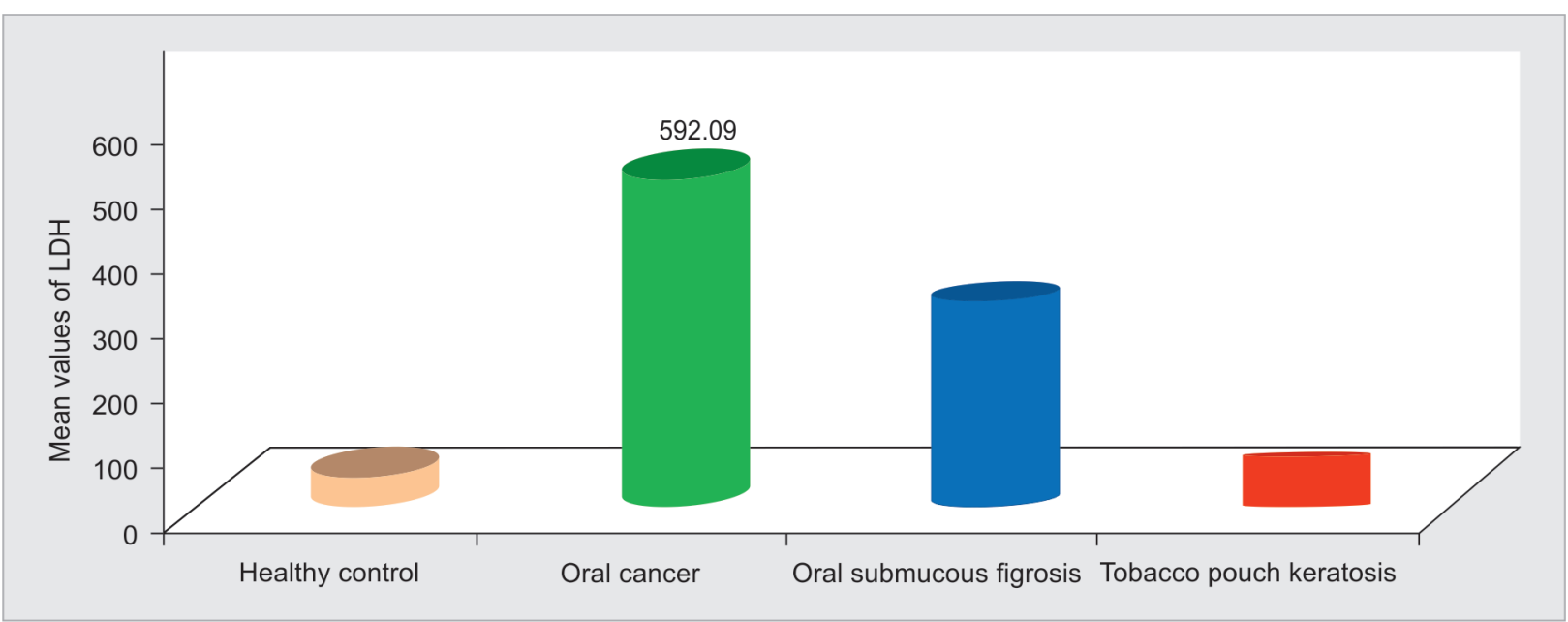

Fig. 1: Comparison of mean values of LDH in the study group and control group

Table 1: Comparison of mean and SD values of LDH IU/L in the healthy control group, oral cancer group, oral submucous fibrosis group, and habitual tobacco chewers group

\begin{tabular}{llll}
\hline $\begin{array}{l}\text { Healthy control } \\
(n=30)\end{array}$ & $\begin{array}{l}\text { Oral cancer } \\
(n=30)\end{array}$ & $\begin{array}{l}\text { Oral sub- } \\
\text { mucous fibrosis } \\
(n=30)\end{array}$ & $\begin{array}{l}\text { Habitual } \\
\text { tobacco chewers } \\
(n=30)\end{array}$ \\
Mean $\pm S D$ & Mean $\pm S D$ & Mean $\pm S D$ & Mean $\pm S D$ \\
\hline $86.12 \pm 7.05$ & $592.09 \pm 28.57$ & $350.43 \pm 5.90$ & $125.19 \pm 13.42$ \\
\hline
\end{tabular}

Table 2: One-way ANOVA test with values of $F=6056.2, p<0.001$, which is highly significant

\begin{tabular}{lcll}
\hline Source of variation & $\begin{array}{l}\text { Degrees of } \\
\text { freedom (d.f.) }\end{array}$ & Sum of squares & Mean square \\
\hline $\begin{array}{l}\text { Treatments } \\
\text { (between columns) }\end{array}$ & 3 & $4,908,860$ & $1,636,287$ \\
$\begin{array}{l}\text { Residuals (within } \\
\text { columns) }\end{array}$ & 116 & 31,341 & 270.18 \\
\begin{tabular}{l} 
Total \\
\hline
\end{tabular} & 119 & $4,940,202$ & \\
\hline
\end{tabular}

In this study, patients satisfying the selection criteria (inclusion and exclusion) were grouped into four groups namely healthy control, habitual tobacco chewers, oral submucus fibrosis (OSMF), and oral cancer depending upon the histopathological diagnosis.

We found the salivary LDH to be the lowest in healthy controls and highest in oral cancer patients. The reason for increased levels in the study groups may be the chronic nature of the disease etiology along with other associated factors like tobacco and genetic predisposition.

There is demonstration of comparison of mean salivary LDH between all the study groups which had highly significant values $(p<0.001)$. The mean salivary LDH value was consistently higher in oral precancer and was paramount in oral cancer patients.

In their study Shetty et al. ${ }^{12}$ observed the changes in salivary $\mathrm{LDH}$ in healthy control, oral leukoplakia and oral cancer. The levels in the control group, oral leukoplakia group and oral cancer were $79.50 \pm 4.67 \mathrm{IU} / \mathrm{L}, 136.46 \pm 3.36 \mathrm{IU} / \mathrm{L}$ and $148.77 \pm 4.83 \mathrm{IU} / \mathrm{L}$, respectively, which demonstrated significant discrepancy in the mean salivary levels of the above groups. The $p$ value was 0.001 in all the three groups. It was observed that salivary LDH levels were systematically higher in oral precancer and cancer patients.
The results of our study show significant correlation with the above study.

Merza et al. ${ }^{13}$ measured the levels of $L D H$, alkaline phosphatase enzymes and immunoglobulins in serum and saliva, for acute leukemia $(A L)$ patients, OSCC patients and apparently healthy individuals as the control group. As per the outcome of this research, serum and saliva enzymes showed a significant increase in LDH, salivary levels with $p<0.05$ levels of AL and OSCC in comparison to the control group. These observations are also summarized in our study.

Divyalaxmi et al. ${ }^{14}$ conducted study in order to find out the significance of $\mathrm{LDH}$, alkaline phosphatase in salivary samples of oral leukoplakia patients, oral squamous cell carcinoma patients and control groups, in order to assess whether estimation of these markers in leukoplakia is valuable in assessing the malignant risk potential. The mean values for $\mathrm{LDH}$ were found to be about $79.70 \mathrm{IU} / \mathrm{L}$, $102.54 \mathrm{IU} / \mathrm{L}$ and $268.57 \mathrm{IU} / \mathrm{L}$ for control, oral leukoplakia and oral squamous cell carcinoma, respectively. They had $p$ value $<0.001$ for $\mathrm{LDH}$ and have significant difference in the values in healthy control and oral cancer, which is analogous to the present study.

Comprehensive salivary analysis was performed to evaluate biochemical parameters in the saliva of oral squamous cell carcinoma patients and healthy and age- and gender-matched individuals by Shipitzer et al., ${ }^{15}$ statistical analysis showed that there was an $88 \%$ increase in the levels of salivary LDH in oral squamous cell carcinoma patients when compared to the control group which was highly significant $(p=0.002)$. Our study showed some resemblance in the value of $p<0.001$ when compared with healthy controls and oral cancer patients.

Similar findings were made by Shpitzer et al. ${ }^{16}$ They performed the study among patients with tongue cancer. They measured the levels of eight salivary markers akin to oxidative stress, DNA repair, carcinogenesis, metastasis, cellular proliferation and death. Of the eight markers, five have increased in cancer patients by 39-246\%: carbonyls, LDH, metalloproteinase-9, Ki67 and Cyclin D1 as compared to controls ( $p \leq 0.01$ ). The value for salivary LDH was $p=0.002$.

The study by Sivaramakrishnan et al. ${ }^{17}$ assessed the LDH enzyme activity in saliva and serum of OSMF patients. Sixty subjects were recruited for this study and divided into two groups, 30 subjects with OSMF (group A) and 30 healthy controls (group B). The average 
salivary LDH value for group A was $606.83 \pm 60.09 \mathrm{U} / \mathrm{L}$ and for group $B$ it was $80.73 \pm 20.06 \mathrm{U} / \mathrm{L}$. The salivary LDH was greater in group $A$ than in group $B$ and this was statistically significant and similar to our study.

$\mathrm{LDH}$ is present in the cytoplasm of all normal living cells and is considered as a metabolic enzyme which becomes extracellular upon cell death. In oral precancerous and cancer patients, its presence is consistently at a higher level when compared with normal patients. This could be due to the extracellular presence, which is always related to cell necrosis and tissue breakdown. ${ }^{18}$

Salivary diagnosis helps in incipient detection of oral cancer and could be used as a screening tool when contemporaneous analysis for significantly increased markers is carried out. As a consequence, salivary LDH deserves more attention by researchers.

Definitive diagnosis is always based on histopathological examinations which remain as the gold standard.

\section{ConcLusion}

Thus it can be concluded that salivary LDH increased in all the study groups. The profile of salivary LDH was found to have an entirely different pattern from that found in plasma, similar to that found in oral epithelium, indicating that the major source of salivary LDH is probably the oral epithelium-shedding cells. Salivary LDH may be used as a diagnostic tool for early cancer diagnosis, prognosis, postoperative monitoring and mass population screening. Thus, the present study was conducted on salivary LDH estimation in various groups as it is a simple, easily performable, noninvasive procedure and easily accepted by the patient. Hence, it can prove to be a valuable substitute to other invasive and costly diagnostic modalities.

\section{Clinical significance}

Being a simple, noninvasive procedure and easily accepted by patients, salivary LDH estimation can prove to be a valuable substitute for other invasive and costly diagnostic modalities as a biochemical marker.

\section{References}

1. Nagler RM, Lischinsky S, et al. New insight salivary lactate dehydrogenase of human subjects. J Lab Clin Med 2001;137(5):363-369. DOI: $10.1067 / \mathrm{mlc} .2001 .114710$.

2. Massano J, Regateiro FS, et al. Oral squamous cell carcinoma: review of prognostic and predictive factors. Oral Surg Oral Med Oral Pathol Oral Radiol Endod 2006;102:67-76. DOI: 10.1016/j.tripleo.2005.07.038.
3. Greenberg MS, Glick, M, et al. Burkets Oral Medicine, 11th ed., Elsevier publication; 2008.

4. Carnelio S, Gabriel S, et al. A Brief Review of Common Oral Premalignant Lesions with Emphasis on Their Management and Cancer Prevention. Indian J Surg Aug 2011;73:256-261. DOI: 10.1007/ s12262-011-0286-6.

5. Shpitzer T, Hamzany Y, et al. Salivary analysis of oral cancer biomarkers. Br J Cancer 2009;101:1194-1198. DOI: 10.1038/sj.bjc.6605290.

6. Cheng B, Rhodus NL, et al. Detection of apoptotic cells in whole saliva of patients with oral premalignant and malignat lesions: A preliminary study. Oral Surg Oral Med Oral Pathol Oral Radiol Endod 2004;97:465-470. DOI: 10.1016/j.tripleo.2003.12.020.

7. Denny YP, Ho CM. The oral fluid MEMS/NEMS Chips (OFMNC): diagnostic and translational application. Adv Dent Res 2005;18:3-5. DOI: 10.1177/154407370501800102.

8. Bigler LR, Streckfus CF, et al. Salivary biomarkers for the detection of malignant tumors that are remote from the oral cavity. Clin Lab Med 2009;29:71-85. DOI: 10.1016/j.cll.2009.01.004.

9. Pundir S, Saxena S, et al. Oral submucous fibrosis a disease with malignant potential-Report of two cases. J Clin Exp Dent 2010;2: 215-218. DOI: 10.4317/jced.2.e215.

10. Epstein JB. Oral cancer. In: Greenberg M, Glick M. ed. Oral Medicine, Diagnosis and Treatment, 10th ed. India: Elsevier; 2003. pp. 194-234.

11. Pelicano H, Martin DS, et al. Glycolysis inhibition for anticancer treatment. Oncogene 2006;25:4633-4646. DOI: 10.1038/sj.onc. 1209597.

12. Shetty SR, Chadha R, et al. Salivary lactate dehydrogenase levels in oral leukoplakia and oral squamous cell carcinoma: A biochemical and clinicopathological study. J Can Res Ther 2012;8:123-125. DOI: 10.4103/0973-1482.95191.

13. Merza KS, Alaaraji SB, et al. Comparative study on Lactate dehydrogenase, Alkaline phosphatase and Immunoglobulins in serum and saliva of Acute leukemia and oral squamous cell carcinoma patients. Iraqi J Sci 2010;51(2):262-270.

14. Divyalaxmi M, Uma Maheswari TN, et al. Expression of Salivary Biomarkers - Alkaline Phosphatase and Lactate Dehydrogenase in Oral Leukoplakia. Int J ChemTech Res 2014;6:2755-2759.

15. Shipitzer T, Bahar G, et al. A comprehensive salivary analysis for oral cancer diagnosis. J Cancer Res ClinOncol 2007;133:613-617. DOI: 10.1007/s00432-007-0207-z.

16. Shpitzer T, Hamzany Y, et al. Salivary analysis of oral cancer biomarkers. Br J Cancer 2009;101:1194-1198. DOI: 10.1038/sj.bjc.6605290.

17. Sivaramakrishnan M, Sivapathasundharam B, et al. Evaluation of lactate dehydrogenase enzyme activity in saliva and serum of oral submucous fibrosis patients. J Oral Pathol Med 2015 Jul;44(6): 449-452. DOI: 10.1111/jop.12246.

18. Lokesh K, Kannabiran J, et al. Salivary Lactate Dehydrogenase (LDH)- A Novel Technique in Oral Cancer Detection and Diagnosis. J Clin Diagn Res 2016 Feb;10(2):ZC34-ZC37. DOI: 10.7860/JCDR/2016/16243.7223. 